\title{
INFLUENCES OF URBAN EXPANSION ON CULTIVATED LANDS IN CHINA SINCE 1970S
}

\author{
Fang Liu ${ }^{1}$, Zengxiang Zhang ${ }^{1}$, Xiaoli Zhao ${ }^{1}$, Sisi Yu ${ }^{1,2^{*}}$, Wang Xiao ${ }^{1}$, Zuo Lijun ${ }^{1}$ \\ 1 Institute of Remote Sensing and Digital Earth, Chinese Academy of Sciences, Beijing 100101, China - (liufang, zhangzx, zhaoxl, \\ yuss2017, zuolj, wangxiao98)@ radi.ac.cn \\ ${ }^{2}$ University of Chinese Academy of Sciences, Beijing 100049, China
}

KEY WORDS: Urban Expansion, Cultivated Lands, Spatiotemporal Characteristics, Long Timeframe, Remote Sensing, Basic Trends

\begin{abstract}
:
Urban expansion has far-reaching influences on cultivated lands, and has a serious effect on grain output and safety. However, relatively little attention has been paid to monitor cultivated land losses through urban expansion over a long timeframe and multi-frequency, especially its differences on national scale systematically. In this work, the characteristics of Chinese cultivated land dynamics were described using annual occupied area per city, contribution rate of cultivated lands to urban expansion and the classification method of basic trend of cultivated land losses. Results indicate that: (1) in the past four decades, large amount of cultivated lands have been occupied during the urban expansion process, and have become the first land source for Chinese urban expansion. (2) Cultivated land loss among municipalities, provincial capitals and other cities was obviously different. The higher of cities' administrative level was, the more obvious of cultivated land loss in these cities appeared, and the earlier of acceleration loss stage of cultivated lands occurred. (3) Cultivated land loss in five population-size cities was unbalanced, representing obviously different loss process and contribution on urban expansion. The bigger of cities' population size was, the more obvious of cultivated land loss in these cities appeared, and the earlier of acceleration loss stage of cultivated lands occurred. (4) Cultivated land losses during urban expansion process were imbalanced in China, and were classified into seven trends. (5) Chinese cultivated land protection has been carried out from the awakening stage to the deep implementation stage.
\end{abstract}

\section{INTRODUCTION}

Urban expansion is the most direct manifestation of urbanization and urban land-use changes (Liu et al., 2005; Liu et al., 2016), and has far-reaching influences on cultivated lands. As the world most populous country, cultivated lands are occupied on a massive scale in the urban expansion process, thereby having a serious effect on grain output and safety (Tan et al., 2005). Although influences of urban expansion on cultivated lands have been widely and vigorously studied (Tan et al., 2005; Liu et al., 2015; Sun and Zhou, 2016), relatively little attention has been paid to monitor cultivated land losses through urban expansion over a long timeframe and multi-frequency, especially its differences on national scale systematically. In particular, few quantitative indicators about the relationship of coupling development between urban expansion and cultivated land loss have been developed. Additionally, urban expansion always contradicted the smooth and reasonable implementation of cultivated land protection policies; however, evaluations about the implementation effects of cultivated land protection policies have not been fully elucidated.

This study aims to rebuild the impact of urban expansion on cultivated land losses of 75 main cities in China using the visual interpretation method based on multi-source remotely sensed imagery during the period from 1970s-2017, recognize the overall characteristics of Chinese cultivated land losses, analyze the differences in cultivated land loss from the aspects of administrative levels and population sizes, distinguish different trends for cultivated land losses, disclose diverse stages for cultivated land protection undergoing, provide scientific data to support cultivated land protection, urban planning and construction in China, and indicate the direction for the sustainable and healthy development of urban lands.

\section{MAIN BODY}

\subsection{Study Area}

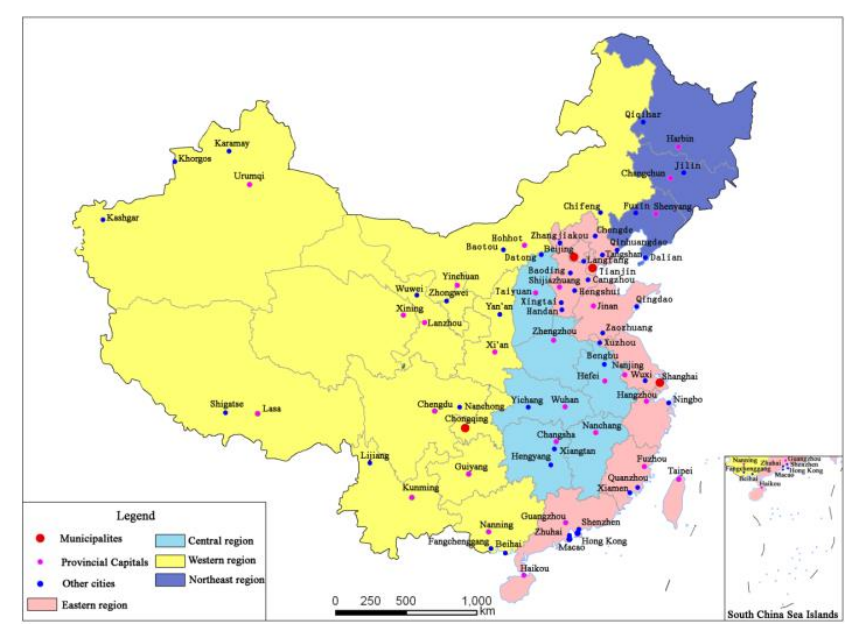

Figure 1 Spatial distribution of 75 sample cities in China

Based on city location, administrative level, city population and remotely sensed imagery availability, 75 cities are selected as sample cities in this work, including 4 municipalities, 28

\footnotetext{
${ }^{*}$ Corresponding author
} 
provincial capitals, 2 special administrative region, and 41 prefectural-level cities and below (named "other cities" thereafter) (Figure 1). These cities only cover $2.85 \%$ territory of China, but hold $35.30 \%$ population and load $54.74 \%$ social economic activities (MOHURD, 2015). Most of these cities are situated in plain areas surrounded by cultivated lands with rich water resources and suitable lands for agriculture. Land expansion of these cities is often associated with a loss of cultivated land. In this work, cultivated land refers to land under permanent crops, temporary crops, land under market and kitchen gardens and temporarily fallow land, etc., but not including meadow and pasture. We only pay attention to cultivated land losses around the core built-up areas, where buildings have been developed contiguously, and where municipal utilities and public facilities are available (MOHURD, 1999).

\subsection{Materials and Pre-processing}

Totally, 1558 scenes of multi-source remotely sensed imagery were used in this study (Zhang et al., 2014a), including 151 scenes of Landsat Multi- Spectral Scanner (MSS) (during the time periods between 1970s and 1980s), 849 scenes of Landsat Thematic Mapper or Enhanced Thematic Mapper Plus (TM/ ETM+) imagery (1984-2011), 67 scenes of China-Brazil Earth Resources Satellite (CBERS) Charge-coupled Device (CCD) imagery (2000-2009), 129 scenes of Environmental Satellite (HJ-1) CCD imagery (2010-2013) and 362 scenes of Landsat Operational Land Imager (OLI) imagery (2013-2016). All these imagery have the time phase of vigorous vegetation growth and less than $10 \%$ cloud amount, and their spatial resolutions range from $19.5 \mathrm{~m}$ to $80 \mathrm{~m}$.

In this work, cultivated lands include paddy fields and dry lands on the basis of the National Land Use/cover Database of China (NLUD-C, hereafter). Man-machine interactive visual interpretation method was employed to monitor cultivated land loss during urban expansion process. To facilitate interpreting imagery, pre-processing of multi-source imagery were operated with the same processing procedure, including band composition using standard false-color synthesis, imagery enhancement adopting linear contrast stretching and histogram equalization (Liu et al., 2012), and imagery rectification based on 1:100,000 topographic maps of China. After these pre-processing steps, all imagery was rectified to a common ALBERS coordinate system with geometric rectification errors for the same feature point less than two pixels. To guarantee the interpretation accuracy, mapping standards of the National Land Use Change Database of China (NLUD-C) were adopted to monitor cultivated land losses resulting from urban expansion. Specific standards are as follows: 1) Visual interpretation is operated based on professional knowledge and experience; 2) in the $1: 100,000$ scale mapping process, only patches larger than $2 \mathrm{~mm} \times 2 \mathrm{~mm}$, which is equivalent to $200 \mathrm{~m}$ $\times 200 \mathrm{~m}$ urban lands, can be mapped; 3) location deviation of feature interpretation is controlled to less than $0.5 \mathrm{~mm}$; 4) during the interpretation process, field verification in the sample positions and some auxiliary materials (such as the Google Earth platform and topographic maps) must be referred to, and interpretation accuracy should be higher than $90 \%$ (Zhang et al., 2012; Shi et al., 2015). Taking municipalities for example, Figure 2 represented the final impact of urban expansion on cultivated lands in the past four decades with high-frequency. All imagery pro-processing steps and monitoring processes were performed on the Modular GIS Environment (MGE) platform. After interpretation, cultivated land losses information was edited on the Arc/Info (version
10.2) platform. Totally, 1432 phases of cultivated land loss data were finished.

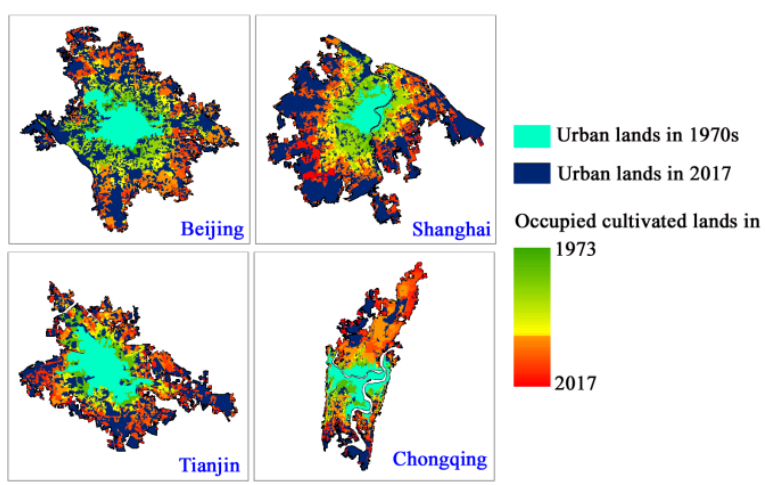

Figure 2 the impact of urban expansion on cultivated lands in 1970s-2017

\subsection{Methodology}

2.3.1 Annual occupied area per city: To describe cultivated land losses caused by urban expansion and eliminate the impacts of different sample numbers on cultivated land losses (Liu et al, 2016), annual occupied area per city (AOAC) was used in this study.

$$
\mathrm{AOAC}=\frac{\sum_{\mathrm{i}=1}^{\mathrm{N}} \frac{\mathrm{CL}_{\mathrm{i}(\mathrm{t}+\mathrm{n})}-\mathrm{CL}_{\mathrm{i}(\mathrm{t})}}{\mathrm{n}}}{\mathrm{N}}
$$

Where $A O A C$ is the annual occupied area per city during $\mathrm{t}-\mathrm{t}+\mathrm{n}$; $C L_{i(t+n)}$ and $C L_{i(t)}$ are the occupied areas of cultivated lands by urban expansion in city $i$ at time $t+n$ and $t$, respectively; $n$ is the interval of the calculation period (in years); and $N$ is the city number of the research region.

2.3.2 Contribution rate: To describe the effect of urban expansion on cultivated and evaluate spatio-temporal characteristics of cultivated land losses resulting from urban expansion, a new index named contribution rate $(C R)$ was established, and it is described as:

$$
\mathrm{CR}=\frac{\mathrm{CL}_{\mathrm{t} \rightarrow \mathrm{t}+\mathrm{n}}}{\mathrm{U}_{\mathrm{t}+\mathrm{n}}-\mathrm{U}_{\mathrm{t}}} \times 100 \%
$$

where $C R(\%)$ is the contribution rate of cultivated lands to urban expansion; $\mathrm{CL}_{\mathrm{t} \rightarrow \mathrm{t}+\mathrm{n}}$ is the area of cultivated lands converted to urban lands during $t-t+n ; U_{t+n}$ and $U_{t}$ are the areas of urban lands at time $t+n$ and $\mathrm{t}$; and $n$ is the interval of the calculation period (in years).

2.3.3 Classification of basic trend of cultivated land losses: To classify the basic trends of cultivated land losses resulting from urban expansion, Person correlation coefficient was employed in this study. Firstly, min-max normalization method was used to normalize the original values $(A O A C$ or $C R)$ in different years (Equation (3) and Equation (4)). Then, Person correlation of normalized values of two samples was calculated.

$$
\begin{aligned}
& x_{i}^{\prime}=\frac{x_{i}-x_{\min }}{x_{\max }-x_{\min }} \\
& y_{i}^{\prime}=\frac{y_{i}-y_{\min }}{y_{\max }-y_{\min }}
\end{aligned}
$$




$$
r_{x y}=\frac{\sum_{i=1}^{n}\left(x_{i}^{\prime}-\bar{x}\right)\left(y_{i}^{\prime}-\bar{y}\right)}{\sqrt{\sum_{i=1}^{n}\left(x_{i}^{\prime}-\bar{x}\right)^{2} \sum_{i=1}^{n}\left(y_{i}^{\prime}-\bar{y}\right)^{2}}}
$$

where $\mathrm{x}_{\mathrm{i}}^{\prime}$ and $\mathrm{y}_{\mathrm{i}}^{\prime}$ indicate the normalization value of $\mathrm{x}_{\mathrm{i}}$ and $\mathrm{y}_{\mathrm{i}} ; \mathrm{x}_{\mathrm{i}}, \mathrm{x}_{\max }, \mathrm{x}_{\min }$, and $\overline{\mathrm{x}}$ donate the $i$ th original value $(A O A C$ or $C R$ ), the maximum value, the minimum value and the normalization mean of sample $\mathrm{x}$, respectively; $\mathrm{y}_{\mathrm{i}}, \mathrm{y}_{\max }, \mathrm{y}_{\min }$, and $\bar{y}$ mean the $i$ th original value, the maximum value, the minimum value and the normalization mean of sample $y$ correspondingly; $r_{x y}$ is Pearson correlation coefficient of sample $\mathrm{x}$ and sample $\mathrm{y}$.

After these two steps mentioned above, correlation coefficients between any two cities were calculated from 1970s to 2017. Two $75 \times 75$ correlation coefficient matrixes were obtained, reflecting similarity degrees of $A O A C$ and $C R$ between any two cities. For each city, the most similar city was selected by selecting the maximum correlation coefficient and two 75 pairs of city groups were thereby formed, respectively. The more-related pairs of cities were classified into one group.

\subsection{Result}

2.4.1 Overall characteristic of cultivated losses during the urban expansion process: Since the 1970s, cultivated land has become the first land source for rapid urban expansion with a CR of $54.95 \%$. A total of $11151.77 \mathrm{~km}^{2}$ of cultivated lands in 75 cities have been occupied in the urban expansion process with an average of $148.69 \mathrm{~km}^{2}$ per city. With the ongoing industrialization and urbanization process, the speed of cultivated land losses increased in a fluctuating manner with the $A O A E$ enhancing from $0.61 \mathrm{~km}^{2}$ in the 1970 s to $4.58 \mathrm{~km}^{2}$ in 2017 (Figure 3), and even reached the peak value of $8.74 \mathrm{~km}^{2}$ in 2011. Though the speed of cultivated land losses increased on the whole, powerful guidance of policies about cultivated land protection has had profound influences on controlling cultivated land losses since the 1970s. Before the end of the 1980s, authoritative departments, laws and regulations on cultivated land protection were all lacking, directly resulting to the high contribution of cultivated lands on urban expansion. Until the end of the 1980s, the decentralized land management of China was broken after the establishment of the land administration in 1986, and the $C R$ of cultivated lands on urban expansion decreased dramatically from $68.08 \%$ in 1987 to $44.98 \%$ in 2017.

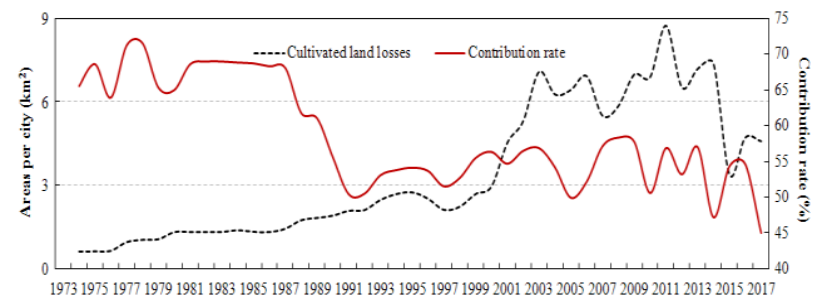

Figure 3. Cultivated land losses during 1970s-2017

2.4.2 Differences of cultivated land loss in Chinese urban expansion process: Cultivated land loss in China is uneven. To synthetically reveal cultivated land loss and provide scientific data for evaluating cultivated land protection among various types of cities, differences of cultivated land loss were analyzed from administrative level and population size aspects. From the aspect of administrative levels, 75 sample cities were classified into municipalities (4 cities), provincial capitals (30cities, including 2 special administrative regions) and other cities (41 cities, including prefectural-level cities and below). Referring to the latest Chinese city scale classification criterion, all monitored cities in this work were grouped as five-level population sizes based on the urban population in 2015 (MOHURD, 2015), including super megacities (4 cities), megacities (10 cities), large cities (42 cities), medium-sized cities (12 cities) and small cities (7cities).

2.4.3 Differences of cultivated land loss in China: Cultivated land loss in municipalities was most evident (Figure 4) with largest averaged loss area, the rapidest loss speed, and the biggest contribution rate on urban expansion. Totally 2173.76 $\mathrm{km}^{2}$ of cultivated lands in four municipalities have been occupied in the urban expansion process, with an average of $543.44 \mathrm{~km}^{2}$ per city. Cultivated lands in municipalities lost the most rapidly with the average $A O A E$ high to $12.53 \mathrm{~km}^{2}, 3.72$ times of the national average. The loss speed exhibited a significant fluctuating trend of growth with the $A O A E$ increasing from $2.21 \mathrm{~km}^{2}$ in the $1970 \mathrm{~s}$ to $13.96 \mathrm{~km}^{2}$ in 2017 . Due to the obvious urban expansion process in municipalities, cultivated land loss underwent one relative stable and slow stage (before 1987), three acceleration stages (1987-1998, 2000-2003, and 2007-2009) and two deceleration stages (1998-2000 and after 2009). Contribution of cultivated lands on urban expansion exhibited a high contribution rate $(61.20 \%)$ and an obvious fluctuating trend in the past four decades. Cultivated land loss in provincial capitals was less serious than that in municipalities but much severer than that in other cities, with the averaged loss area, loss speed, and contribution rate on urban expansion all higher than the national average. The averaged loss area of cultivated lands in provincial capitals was $200.54 \mathrm{~km}^{2}$ per city, which was 2.78 times of that in other cities, but only $36.90 \%$ of that in municipalities. Annual cultivated lands lost at an average speed of $4.58 \mathrm{~km}^{2}$ per city, which was only $36.57 \%$ of that in municipalities. Cultivated land loss in provincial capitals had the similar trend to the national trend, either its loss speed or its contribution on urban expansion. Although other cities accounted for $54.67 \%$ of 75 sample cities, their cultivated land loss was the most unobvious with the smallest averaged loss area, the slowest loss speed, and the smallest contribution rate on urban expansion. The averaged loss area of cultivated lands in other cities was only $72.24 \mathrm{~km}^{2}$ per city, far smaller than the national level. Affected by the small city size of other cities, annual loss speed of cultivated lands in other cities was $1.62 \mathrm{~km}^{2}$ per city, only $12.91 \%$ and $35.30 \%$ of that in municipalities and provincial capitals. The loss speed of cultivated lands in other cities also had the similar trend to the national trend with an increase from $0.06 \mathrm{~km}^{2}$ in the 1970 s to 2.07 in 2017 . The CR of other cities ranged from $35.93 \%$ to $92.84 \%$, and fluctuated much more obviously than other two types of cities.
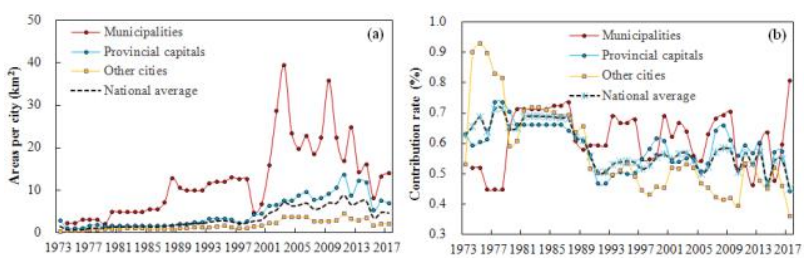

Figure 4. Cultivated land losses in different administrative-level cities (a) annual occupied area per city; (b) contribution rate 
Among five groups of cities with different population sizes (Figure 5), cultivated land loss in super megacities and megacities were more significant. Loss speed in these two city groups were much higher than the national average, with the averaged loss areas of $562.10 \mathrm{~km}^{2}$ per city and $312.11 \mathrm{~km}^{2}$ per city, and the annual loss speeds of $12.83 \mathrm{~km}^{2}$ per city and 7.20 $\mathrm{km}^{2}$ per city, respectively. Fluctuating trends of super megacities and megacities were much more obvious than other three city groups. Obvious acceleration loss of cultivated lands emerged in super megacities in the late of 1980s, while appeared in the mid-1990s in megacities. Furthermore, the peak value of loss speed in super megacities emerged in 2003, but appeared 7 years later in megacities. Contributions of cultivated lands on urban expansion in these two city groups all tended to increase in the past four decades, which was opposite to that in other three city groups. Though large cities included the largest number of cities, their cultivated land losses both in loss process and its influences on urban expansion, were less obvious than that in super megacities and megacities. Since the 1970 s, cultivated land loss of large cities was only $124.31 \mathrm{~km}^{2}$ per city, only accounting for $22.11 \%$ and $39.83 \%$ of that in super megacities and megacities. The loss speed of large cities was much slower than that in megacities and super megacities, with the $A O A C$ of $2.85 \mathrm{~km}^{2}$. The loss speed and contribution rate all represented high similar fluctuating trends to the national trends. Obvious acceleration of cultivated land loss in large cities was posterior to that in super megacities and megacities but prior to that in medium-sized cities and small cities. The average CR of large cities was $54.02 \%$, slightly lower than the national level. Cultivated land losses in medium-sized cities and small cities were unobvious. Before the 21 st century, loss speeds of these two city groups were relatively stable, and their peak values all appeared in 2013. Additionally, significant acceleration loss of cultivated lands emerged in these two city groups all posterior to that in other three city groups. Similar to large cities, the CR of medium-sized and small cities all tended to decrease in the past four decades. Though the CR IN the medium-sized cities was approximate to the national average while much smaller in small cities than the national level, their CRs were all much more fluctuated than other three city groups.
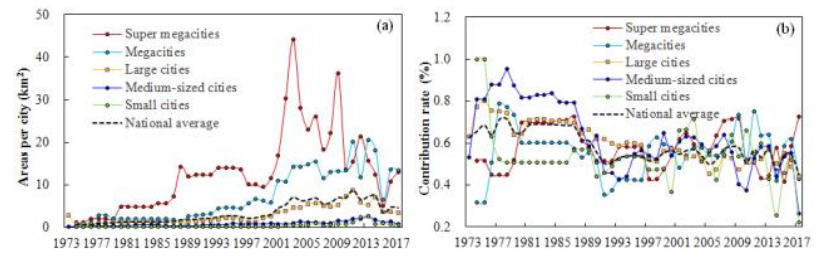

Figure 5. Cultivated land losses in different population-size cities (a) annual occupied area per city; (b) contribution rate

2.4.4 Basic trends of cultivated losses during the urban expansion process: In the past four decades, cultivated land losses during urban expansion process were imbalanced in China with the correlation coefficients of cultivated land loss speed between every sample city and the national average ranging from -0.67 to 0.89 and the correlation coefficients of cultivated land contribution to urban expansion between every city and the national average fluctuating from -0.62 and 0.81 (Figure 6).

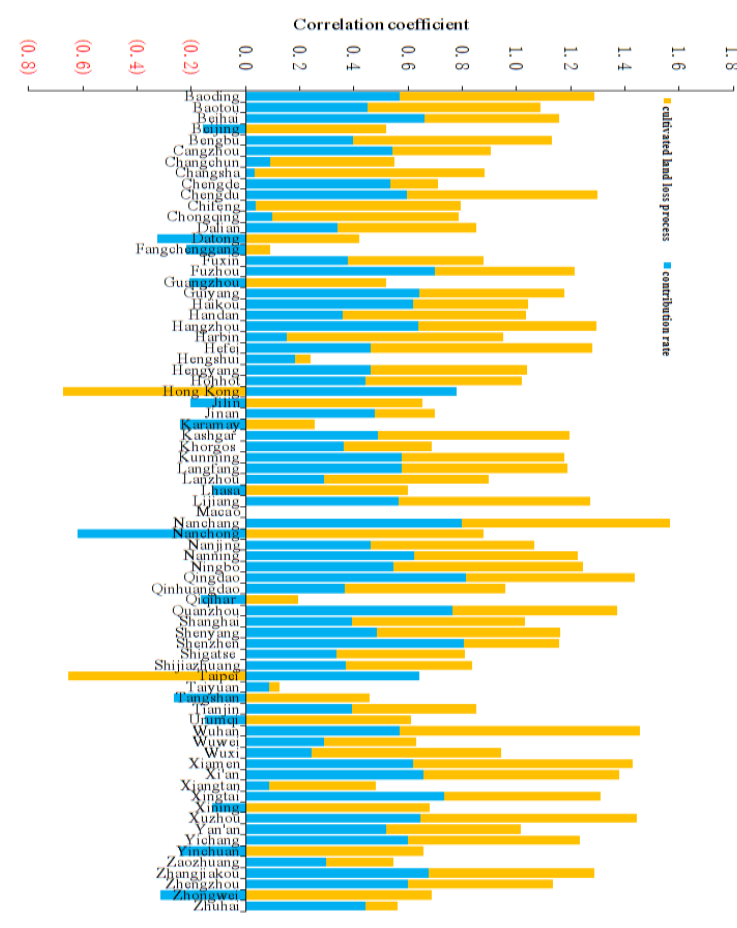

Figure 6 Correlation coefficients of cultivated land loss speed between 75 cities and the national average (in yellow bar) and cultivated land contribution to urban expansion between 75 cities and the national average (in blue bar).

Based on obvious different loss speed and contribution rate, Chinese cultivated land losses during urban expansion process were classified into seven trends, A) accelerating first and then decelerating for loss speed with sustained decrease of contribution rate; $\mathrm{B}$ ) accelerating first and then decelerating for loss speed with continued increase of contribution rate; C) accelerating first and then decelerating for both loss speed and contribution rate; D) accelerating first and then decelerating for loss speed with opposite trend for contribution rate; E) sustained acceleration for loss speed with continued decrease for contribution rate; F) sustained acceleration for loss speed with contribution rate decreasing first and then increasing; and G) decreasing first and then increasing for both loss speed and contribution rate (Figure 7). 


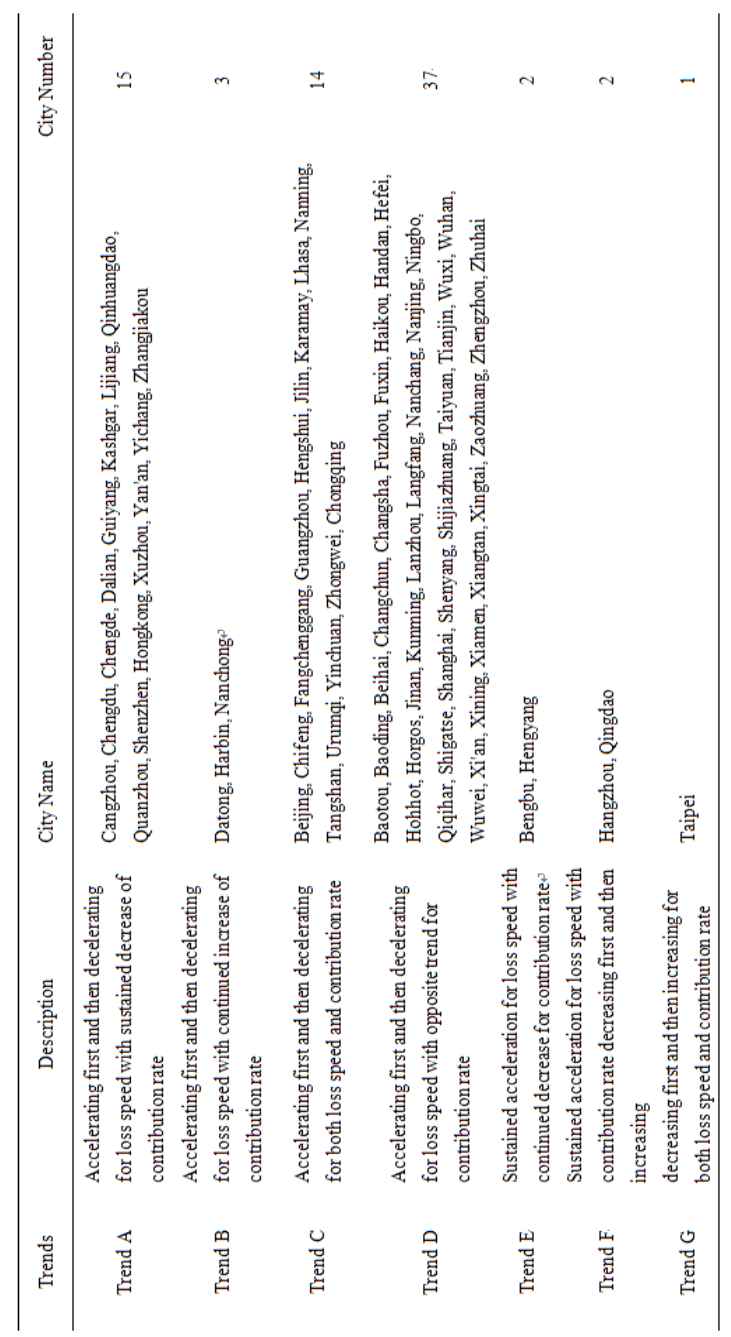

Figure 7 Basic trends classification of cultivated losses among 75 sample cities

2.4.5 Evaluation of cultivated land protection in China: With vast population and scarce cultivated land per capita, cultivated land conservation/ food security has been acknowledged as one of the main factors affecting the sustainable socio-economic development in China. In the urban expansion process of China, cultivated land plays an important role and has become the primary land source. Cultivated land loss and urban expansion among 75 sample cities had a well linear relationship with a high correlation coefficient of 0.88 , which indicated that cultivated land loss in China was directly impacted by urban expansion. Under the powerful guidance of policies, urban expansion in China was obvious and went through different stages, which had a similar trend to cultivated land losses during this process. Since the implementation of the reform and opening-up policy in the late 1970s, a series of policies, strategies and planning about cultivated land protection have been issued to control the occupation of cultivated land by urban lands, and cultivated land protection underwent the awakening stage, initial stage, uncontrolled stage, effectively carrying out stage, and deep implementation stage in 1970s-1985, 1985-1997, 1997-2003, 2003-2011, and after 2011, respectively.
Based on 1558 scenes of multi-source remotely sensed imagery, the occupation of Chinese urban expansion on cultivated lands in the past four decades with high-frequency were reconstructed by employing the visual interpretation method. Annual occupied area per city and contribution rate of cultivated lands to urban expansion were used to describe cultivated land losses in China and its differences, and classification of basic trend of cultivated land losses were built mainly on the basis of Person correlation coefficient to distinguish different trends for Chinese cultivated land losses during the urban expansion process. The conclusions are as follows:

(1) Since 1970s, cultivated land lost dramatically and has become the first land source for Chinese urban expansion with a total loss of $11151.77 \mathrm{~km}^{2}$ in 75 sample cities and a CR of $54.95 \%$. Similar to the urban expansion process, the speed of cultivated land losses also underwent a fluctuating process, and the $\mathrm{CR}$ of cultivated lands on urban expansion decreasing dramatically.

(2) In the past four decades, the loss speed in municipalities, provincial capitals, and other cities all increased fluctuantly. Municipalities had the largest averaged cultivated land loss area, the rapidest loss speed, and the biggest contribution rate on urban expansion, followed by provincial capitals and other cities successively. Contribution rate of cultivated lands on urban expansion in municipalities tended to increase, while decreased in provincial capitals and other cities. The higher of cities' administrative level was, the more obvious of cultivated land loss in these cities appeared, and the earlier of acceleration loss stage of cultivated lands occurred.

(3) Cultivated land loss in five population-size cities was unbalanced, representing obviously different loss process and contribution on urban expansion. The loss speed of five city groups with different population sizes all increased fluctuantly. Contribution rate of cultivated lands on urban expansion in megacities and above tended to increase, whereas decreased in large cities, medium-sized cities and small cities. The bigger of cities' population size was, the more obvious of cultivated land loss in these cities appeared, and the earlier of acceleration loss stage of cultivated lands occurred. The averaged loss area and the loss speed in super megacities were the biggest and the rapidest, followed by megacities, large cities, medium-sized cities and small cities.

(4) Cultivated land losses during urban expansion process were imbalanced in China. Based on its loss speed and contribution rate to urban expansion, cultivated land losses in 75 sample cities were classified into seven trends.

(5) Powerful guidance of policies about cultivated land protection has had profound influences on controlling cultivated land losses since the 1970s. In the past four decades, Chinese cultivated land protection has been carried out from the awakening stage to the deep implementation stage.

\section{ACKNOWLEDGEMENTS}

This research was supported by the International Partnership Program of Chinese Academy of Sciences [grant number 131C11KYSB20160061]. The authors also express appreciation to all persons who kindly pre-process the remotely sensed imagery and interpret the impact of urban expansion on cultivated lands in this work.

\section{CONCLUSIONS}




\section{REFERENCES}

Liu, F., Zhang, Z., Shi, L., Zhao, X., Xu, J., Yi, L., Liu, B., Wen, Q., Hu, S., Wang, X., Zuo, L., Li, N., Li, M., 2016. Urban expansion in China and its spatial-temporal differences over the past four decades. Journal of Geographical Sciences, 26(10), pp. $1477-1496$.

Liu, J., Zhan, J., Deng, X., 2005. Spatio-temporal patterns and driving forces of urban land expansion in China during the economic reform era. AMBIO: A Journal of the Human Environment, 34(6), pp. 450-455.

Liu, J., Zhang, Q., Hu, Y., 2012. Regional differences of China's urban expansion from late 20th to early 21 st century based on remote sensing information. Chinese Geographical Science, 22(1), pp. 1-14.

Liu, T., Liu, H., Qi, Y., 2015. Construction land expansion and cultivated land protection in urbanizing China: Insights from national land surveys, 1996-2006. Habitat International, 46, pp. 13-22.

MOHURD (Ministry of housing and urban-rural development of the people's republic of China), 2015. China urban-rural construction statistical yearbook. Beijing: China Planning Press. (in Chinese)

MOHURD (Ministry of Housing and Urban-Rural Development of the People's Republic of China), 1999. GB/T 50280-98 City Planning Basic Terminology Standards. Beijing: China Building Industry Press. (in Chinese)

Shi, L., Liu, F., Zhang, Z., Zhao, X., Liu, B., Xu, J., Wen, Q., Yi, L., Hu, S., 2015. Spatial differences of coastal urban expansion in China from 1970s to 2013. Chinese Geographical Sciences, 25(4), pp. 389-403.

Sun, B., Zhou, Q., 2016. Expressing the spatio-temporal pattern of farmland change in arid lands using landscape metrics. Journal of arid environments, 124, pp. 118-127.

Tan, M., Li, X., Liu, C., 2005. Urban land expansion and arable land loss of the major cities in China in the 1990s. Science in China Series D-Earth Sciences, 48(9), pp. 1492-1500.

Zhang, Z., Zhao, X., Wang, X., 2012. Chinese land use. Beijing: Star Map Press. (in Chinese) 